\title{
Der Lissabon-Prozess: Warum eine Hightech-Strategie zur Innovationsförderung nicht ausreicht
}

Mit der im Jahr 2000 beschlossenen Lissabon-Agenda wird der Förderung und Etablierung von Hightech auf europäischer Ebene eine zentrale Funktion zugeschrieben. Ziel ist es, Innovationen und damit Wachstum und Beschäftigung in Europa sicherzustellen. Problematisch daran ist, dass die Betrachtung und Förderung nicht-forschungsintensiver Innovationen und anderer Innovationspotenziale, wie beispielsweise spezifische Fähigkeiten und Ressourcen, vernachlässigt werden. Dies ist vor allem für Lowtech-Sektoren wie Metallverarbeitung, Herstellung von Textilen, Papier und Pappe von Bedeutung, die nach wie vor einen hohen ökonomischen Stellenwert hinsichtlich ihrer Beschäftigungs- und Wertschöpfungsquote haben. ${ }^{1}$

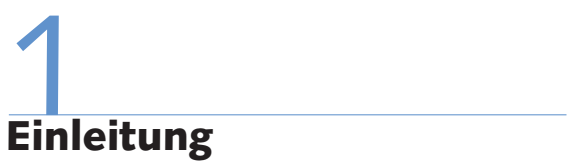

Innovationen gelten nicht nur als ein entscheidender Wettbewerbsfaktor für Unternehmen, sondern auch als treibende Kraft für Wachstum und Beschäftigung in Regionen und Staaten. Das Innovationsspektrum variiert von inkrementellen Veränderungen bis hin zu radikalen Neuerungen bei technischen sowie organisationalen Strukturen, Prozessen und Produkten. Verbunden mit diesem breiten Spektrum an unterschiedlichen Innovationsformen stellt sich die Frage, wie Politik, und hier insbesondere die Politik der Europäischen Union (EU), mit dieser Vielfalt an Innovationsformen und den damit verbundenen Möglichkeiten der Innovationsförderung umgeht. Um sich der Antwort zu nähern, ist zunächst zu klären, welches Verständnis von Innovation in den politisch-programmatischen Dokumenten sowie den wissenschaftlichen Innovationsanalysen der EU vorherrscht. Das beinhaltet die zweite Frage, welche Bedeutung nicht-forschungsintensive, aber innovative, beschäftigungsund wertschöpfungsstarke Sektoren, wie die Herstellung von Kunststoffen und Textilien oder die Metallerzeugung und -verarbeitung, in der innovationspolitischen Programmatik und den Innovationsanalysen auf europäischer Ebene haben.

Die politische Grundlage für die gegenwärtige innovationspolitische Strategie setzte der Europäische Rat im Jahr 2000 mit der Verabschiedung der LissabonAgenda. Als ein zentrales Mittel, um die dort definierten Wachstums- und Beschäftigungsziele zu erreichen, gilt die Steigerung der Innovationstätigkeiten mithilfe eines breit angelegten Sets kohärenter Maßnahmen. Um ein solches Set innovationspolitischer Maßnahmen sowie Indikatoren zur Messung der Innovationsleistung entwickeln zu können, muss definiert sein, was ökonomisch bedeutende Innovationen ausmacht, wodurch sie angeregt und wie sie umgesetzt werden. In Abschnitt 2 wird das Innovationskonzept der EU anhand zentraler Dokumente erörtert, die im Rahmen des Lissabon-Prozesses von unterschiedlichen Organen der EU verfasst worden sind. Deutlich erkennbar ist hier eine starke Verknüpfung von Forschungs- und Innovationspolitik, was eine Fokussierung auf forschungsintensive Innovationen erkennen lässt. Als wissenschaftliche Fundierung der innovationsprogrammatischen Aussagen können die in Abschnitt 3 vorgestellten Erhebungen und Analysen der Innovationsleistung mithilfe von Innovationsindikatoren im Rahmen des Europäischen Innovationsanzeigers bezeichnet werden. Hier wird der Bias auf Forschung und Entwicklung (FuE) als zentraler Innovationsinput aufs Neue deutlich. Basierend auf den Ergebnissen der bisherigen Ausführungen rekonstruiert Abschnitt 4 das Innovationsverständnis auf europäischer Ebene und zeigt, zu welchen Defiziten die Fokussierung auf forschungsintensive Innovationen führt. Abschnitt 5 fasst die Argumentation noch einmal kurz zusammen und formuliert offene Forschungsfragen.

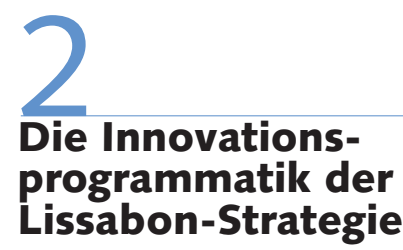

\subsection{DER LISSABON-PROZESS}

"Innovation was recognised to be at the heart of the Lisbon Process" (Europäische Kommission 2004b, S. 7).

Der Europäische Rat legte mit der im Jahr 2000 verabschiedeten Lissabon-Agenda neue strategische Ziele der EU-Politik fest, durch die bis 2010 Wachstum, Beschäftigung und soziale Kohäsion in Europa erhöht werden sollen. Die thematisch umfassende Agenda beinhaltet Strategien in nahezu allen Politikbereichen; Innovationen machte der Europäische Rat zu ei-

\footnotetext{
1 Der vorliegende Beitrag ist die überarbeitete und gekürzte Version eines Vortrags, der im Rahmen der Herbsttagung "Innovation und gesellschaftlicher Wandel " der Sektionen Arbeits- und Industriesoziologie und Wissenschafts- und Technikforschung am 12./13. Oktober 2007 in Dortmund gehalten wurde. Für die hilfreichen Kommentare zu diesem Text möchte ich mich bei Jörg Abel und zwei anonymen Gutachtern bzw. Gutachterinnen bedanken.
}

Katrin Hahn, Dipl. Ök., ist wissenschaftliche Mitarbeiterin am Lehrstuh/ Wirtschafts- und Industriesoziologie der TU Dortmund. Arbeits schwerpunkte: Innovation und Diffusion in industriellen Sektoren, europäische Innovationspolitik.

e-mail: katrin.hahn@tu-dortmund.de 


\section{Abb. 1: Politikfelderübergreifende Innovationspolitik}

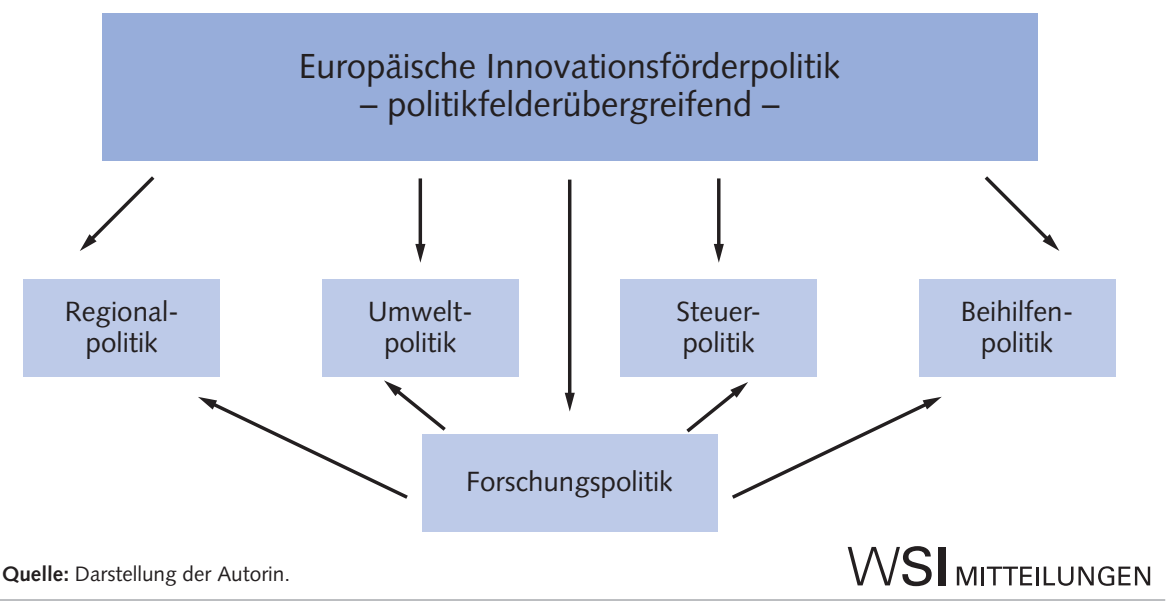

nem der zentralen politischen Themen der Europäischen Union. Die Heranziehung der sogenannten Lissabon-Agenda als Anknüpfungs- und Startpunkt der hier durchgeführten innovationsprogrammatischen Betrachtung fußt auf zwei Ausgangsüberlegungen: Erstens ist der Europäische Rat das höchste europäische Entscheidungsgremium, und zweitens macht die Neuausrichtung der Politik im Jahr 2000 die Lissabon-Agenda zum Sammelbegriff für die allgemein akzeptierten und natürlich auch diskutierten Leitlinien der europäischen Politik bis 2010. Von politisch großer Bedeutung war die Lissabon-Agenda nicht nur für die ständigen europäischen Organe wie die Kommission oder das Parlament, sondern auch für die diversen Stakeholder sowie für die nationalen Regierungen der Mitgliedstaaten.

Die Europäische Kommission (2003a, S. 5) beschreibt in ihrer, ,innovationspolitischen Mitteilung" Innovationen als Neuerungen, die in Wirtschaft und Gesellschaft erfolgreich produziert, angepasst und kommerziell verwertet werden. Als Innovation gilt demnach nicht eine Erfindung oder Neuerung an sich; vielmehr macht erst die erfolgreiche Nutzung und Wertschöpfung im wirtschaftlichen oder/und gesellschaftlichen Kontext eine Erfindung zu einer Innovation. Wie bereits anhand dieser Definition zu sehen ist, präferiert die Kommission ein umfassendes Verständnis von Innovation und daraus resultierend umfassende Möglichkeiten zur Formulierung innovationspolitischer Maßnahmen. Betont wird zunächst die Komplexität von Innovationen, die unter anderem an den unterschiedlichen Möglichkeiten festgemacht wird, Innovationen zu generieren (beispielsweise durch Forschung oder Diffusionsprozesse).

Entsprechend der bereits im Jahr 2004 formulierten Kritik an der Umsetzung der Lissabonner Ziele durch eine hochrangige Sachverständigengruppe unter dem Vorsitz von Wim Kok (Europäische Kommission 2004a) fiel die Halbzeitbilanz der LissabonStrategie ein Jahr später wenig zufriedenstellend aus. Anstelle des erstrebten Anstiegs auf $3 \%$ des Bruttoinlandsprodukts (BIP) (Europäischer Rat 2002, Abs. 47) stagnierten unter anderem die öffentlichen und privaten FuE-Ausgaben. Daraufhin beschließt im März 2005 der Europäische Rat auf der Grundlage des Frühjahrsberichts der Kommission (Europäische Kommission 2005a), die Strategie von Lissabon zu modifizieren und damit neu zu beleben (ebd. S. 3). Die integrierten Leitlinien (Europäische Kommission 2005c) und das Lissabon-Programm der Gemeinschaft (Europäische Kommission 2005b, S. 4), zwei von der Kommission erstellte Strategiepapiere, sollten zur Konkretisierung, Neuformulierung und Abstimmung der politischen Ziele und Aufgaben der Kommission und der Mitgliedstaaten beitragen.

\subsection{POLITIKÜBERGREIFENDE INNOVATIONSPOLITIK}

Betrachtet man nun die unterschiedlichen Maßnahmen aus den verschiedenen politisch-programmatischen Dokumenten genauer, lässt sich das innovationspolitische Maßnahmensample unter den folgenden vier Politikschwerpunkten zusammenfassen:
(1) Erhöhung und Intensivierung von FuEAktivitäten:

- Verbesserung der Koordination von Forschung und Entwicklung in Europa;

- Erhöhung der Mobilität von Forschern; - Mechanismen zur Erhöhung von Investitionen in FuE und spitzentechnologischen Neugründungen - Steuervergünstigungen, Bereitstellung von Risikokapital, Unterstützung durch die Europäische Investitionsbank.

(2) Unterstützung von kleinen und mittelgroßen Unternehmen (KMU):

- Schaffung von Anreizen zur Gründung innovativer KMU, durch die Senkung unternehmerischer Kosten und Bürokratie;

- Förderung von Schlüsselschnittstellen zwischen Unternehmen und Organisationen in Innovationsnetzwerken.

(3) Die Erleichterung des Zugangs zu Risikokapital, vor allem für KMU.

(4) Die Einführung eines kostengünstigen und konkurrenzfähigen gemeinschaftsweiten Gemeinschaftspatents.

Im Zuge der kritischen Auseinandersetzung zum Fortschritt der Lissabon-Strategie $(2004 / 05)$ wurden diese vier Politikbereiche durch staatliche Beihilfen- und Steuerpolitik, Regionalpolitik und Förderung von Ökoinnovationen ergänzt. Auch den öffentlichen Behörden wird über ihr Auftragsvergabewesen Verantwortung bei der Förderung von innovativen Produkten und Dienstleistungen übertragen.

Wie hieraus ersichtlich wird, kann man nur schwer von der Innovationspolitik sprechen. Europäische Innovationspolitik ist durch diese programmatischen Dokumente vielmehr politikfeldübergreifend angelegt. Vor allem über die Forschungsförderung werden verschiedene, innovationsrelevante Politikbereiche miteinander verbunden (Abbildung 1). So werden beispielsweise Maßnahmen aus dem Bereich staatlicher Beihilfen sowie Steuererleichterungen für FuE-Ausgaben (Personal, Kooperationen etc.) vorgeschlagen, um die Erhöhung der FuE-Investitionen zu forcieren (3-\%-Ziel von Barcelona) und somit die Innovationsfähigkeit zu fördern. Innovationskooperationen zwischen Unternehmen und Forschungseinrichtungen sollen gezielt durch regionalpolitische Maßnahmen unterstützt werden. 


\subsection{SCHWERPUNKTE UND DEFIZITE DER INNOVATIONSFÖRDERPOLITIK}

Im Rahmen dieser ressortübergreifenden Innovationsförderpolitik nimmt die Forschungsförderung eine besondere Stellung ein: Forschungsförderung wird gleichzeitig auch als Förderung von Innovationsaktivitäten verstanden. Die Ergebnisse sprechen für ein Verständnis von Innovation auf europäischer Ebene, bei dem Forschung und Entwicklung eine bedeutende Position innerhalb des Innovationsprozesses einnehmen. Die Forschungs- und Innovationspolitik zielt demnach aufgrund der starken Förderung von FuE vor allem auf Unternehmen mit hohen FuE-Aktivitäten $\mathrm{ab}$, wie sie beispielsweise im Biotechnologie- oder Informations- und Kommunikationssektor zu finden sind.

Problematisch an diesem Verständnis sind vor allem zwei Punkte: Erstens werden Innovationspotenziale vernachlässigt, die nicht in unmittelbarem Bezug zu FuE-Aktivitäten stehen. $\mathrm{Zu}$ nennen sind hier spezielle Fähigkeiten, so beispielsweise, schnell und flexibel auf neue technische Marktanforderungen und individuelle Kundenwünsche zu reagieren, Konzepte zu entwickeln, bevor der Kunde sie anfragt, also zukünftige Kundenbedürfnisse oder technische Anforderungen zu antizipieren. Mit dem Konzept der „Innovation Enabling Capabilities" haben Bender/Laestadius (2005) zentrale Fähigkeiten zur Innovationsgenerierung identifiziert, bei denen FuE als alleiniger Innovationstreiber keine (entscheidende) Rolle spielt (vgl. Abschnitt 4). Dazu gehören unter anderem Fähigkeiten, die es ermöglichen, relevantes Wissen zu erkennen, es mit Blick auf den Nutzungskontext zu transformieren und zu erweitern, relevante Akteure zusammenzubringen sowie Artefakte und Wissen zu rekombinieren, um neues Wissen zu erzeugen. Zweitens basiert auch die Förderung von Technologietransfer auf derselben Logik, wonach FuE-Institutionen die Impulsgeber für Innovationsprozesse sind, deren Ergebnisse sich wiederum durch Diffusionsprozesse in weniger forschungsintensive Unternehmen ${ }^{2}$ verbreiten. Diffusion umfasst jedoch mehr als die Verbreitung von Forschungsergebnissen oder den Zukauf von Hightech-Maschinen oder Software durch Lowtech-Unternehmen, wie es in der Kommissionsmitteilung dargestellt wird (Europäische Kommission 2003a, S. 12). Um Diffusionsprozesse erfolgreich umzusetzen, bedarf es spezifischer Fähigkeiten der „Empfängerunternehmen“. Sie müssen die intellektuellen, physischen und finanziellen Kapazitäten bereitstellen, relevante Innovationen identifizieren und auch erfolgreich in den Unternehmensablauf integrieren (Robertson et al. 2005, S. 123).

Der Bias auf Forschungsförderung kann aber nicht nur an der politischen Programmatik gezeigt werden, sondern auch an politiknahen wissenschaftlichen Beschreibungen und Analysen von Innovationen. Dieses Argument wird im Folgenden anhand des europäischen Innovationsanzeigers verdeutlicht.

\section{3 \\ Zur Unvollkommenheit des Indikatorenkonzepts}

Unterschiede zwischen einzelnen Mitgliedstaaten, Wirtschaftssektoren oder der EU im Vergleich zu den USA und Japan können anhand von vergleichenden Innovationsindikatoren aufgezeigt werden. Die Entwicklung eines geeigneten Instruments wurde vom Europäischen Rat bei der Kommission in Auftrag gegeben. ${ }^{3}$ Das Ergebnis ist der Europäische Innovationsanzeiger (European Innovation Scoreboard, EIS), der zum Benchmarking und zum Vergleich der Innovationsleistung vor allem der EUMitgliedstaaten herangezogen wird. So wird mit der Berechnung von nationalen Durchschnittswerten den Mitgliedstaaten eine Vergleichsbasis geliefert, anhand derer Stärken und Schwächen des jeweiligen Staates oder Wirtschaftssektors ablesbar sind (Grupp/Mogee 2004).

Ein Beispiel: Ein Innovationsindikator ist der Anteil der privaten FuE-Aufwendungen am BIP. Im Vergleich zu Japan $(2,4 \%)$ sind die FuE-Aufwendungen in Europa $(1,17 \%)^{4}$ nach Meinung der Experten viel zu gering. Der Europäische Rat in Barcelona beschließt nun eine Erhöhung der Ausgaben für FuE und Innovation auf $3 \%$ des BIP. Die Mitgliedstaaten sind somit angehalten, bis 2010 dieses Ziel voranzutreiben. Mithilfe der Indikatoren des Innovationsanzeigers kann die erfolgreiche Umsetzung des Beschlusses jährlich überprüft werden.

Um die Innovationsleistung zu ermitteln, muss die Auswahl der Indikatoren so zusammengestellt sein, dass sie zum einen
Einflussfaktoren auf Innovationstätigkeiten (Input: Lebenslanges lernen, FuE-Ausgaben u.a.) und zum anderen Ergebnisse des Innovationsprozesses (Output: Patente, Verkauf von Marktneuheiten etc.) erfasst. Hinter der Zusammenstellung der rund 25 Indikatoren existiert also eine Vorstellung davon, wodurch Innovationsprozesse eingeleitet und positiv beeinflusst werden und wie sie ablaufen. ${ }^{5}$ Es zeigt sich, dass ähnlich der Schwerpunktsetzung der politischen Maßnahmen die Auswahl der Indikatoren sehr breit angelegt ist. Um besser nachvollziehen zu können, inwieweit bei dem Europäischen Innovationsanzeiger der Schwerpunkt auf Forschung und Entwicklung gelegt wird, bietet es sich an, die in den Jahren 2001-2007 verwendeten Indikatoren (Europäische Kommission 2001, S. 35ff.; 2002, S. 26; 2003b, S. 2; 2004b, S. 28 f.; 2005d, S. 8 ; 2006, S. 7; 2008, S. 35) nach ihrer Relevanz für die Erfassung von forschungsintensiven Innovationen bis hin $\mathrm{zu}$ allgemeinen Innovationsindikatoren $\mathrm{zu}$ kategorisieren (Übersicht 1):

Die Einordnung der Indikatoren mit direktem Hightech-Bezug ist relativ eindeutig, da dazu natürlich solche Indikatoren wie die Beschäftigung oder die Wertschöpfung in Hightech-Sektoren zählen. Zu den vorrangig hightechrelevanten Indikatoren gehören nicht nur jene wie FuE-Aufwendungen, sondern auch die Patentindikatoren, die auf den ersten Blick als unabhängig von

2 Nicht-forschungsintensive Sektoren werden in der Literatur auch als Lowtech-Sektoren bezeichnet. Darunter fallen entsprechend einer Kategorisierung der OECD Sektoren wie Metallerzeugung und -verarbeitung, Herstellung von Textilien sowie Papier und Pappe. Die Einteilung in die jeweiligen Kategorien wurde anhand von Berechnungen vorgenommen, die auf einem Ranking der FuE-Intensitäten der Jahre 1991-1999 basieren (OECD 2007, S. 219ff.). Demnach gehören zu den Medium-Hightech-Sektoren unter anderem der Maschinen- und Fahrzeugbau, Elektrotechnik und Chemie. Der Hightech-Sektor umfasst u. a. die Pharmazeutische Industrie, den Flugzeugbau sowie Informations- und Kommunikationstechnologien.

3 Im Rahmen des Oslo Manuals der OECD (2005) werden Ansätze zur Entwicklung und Harmonisierung von Innovationserhebungen und -indikatoren aufgeführt.

4 Daten zu den nationalen FuE-Aufwendungen: Europäsche Kommission 2008, S. 16

5 Die Schwierigkeit bei der Entwicklung von Indikatoren liegt nicht nur darin, den Innovationsprozess und die Einflüsse darauf zu identifizieren und zu explizieren, sondern auch darin, dass die Indikatoren quantitativ messbar sein müssen. Dies setzt unter anderem voraus, dass zuverlässige Daten zur Verfügung stehen 


\section{Übersicht 1: Indikatoren des European Innovation Scoreboard (EIS) im Überblick}

\begin{tabular}{|c|c|c|c|c|c|c|c|c|}
\hline & \multicolumn{2}{|c|}{2001} & 02 & 03 & 04 & 05 & 06 & 07 \\
\hline \multicolumn{9}{|c|}{ Indikatoren zur Erfassung von Innovationen im Hightech-Sektor } \\
\hline $\begin{array}{l}\text { 1. Beschäftigung im (Medium-) High-Tech-Sektor / } \\
\text { Verarbeitendes Gewerbe }\end{array}$ & Out1) & $\checkmark$ & $\checkmark$ & $\checkmark$ & $\checkmark$ & $\checkmark$ & $\checkmark$ & $\checkmark$ \\
\hline $\begin{array}{l}\text { 2. Beschäftigung im High-Tech- } \\
\text { Dienstleistungssektor }\end{array}$ & Out & $\checkmark$ & $\checkmark$ & $\checkmark$ & $\checkmark$ & $\checkmark$ & $\checkmark$ & $\checkmark$ \\
\hline 3. Export von High-Tech-Produkten & Out & & & & & $\checkmark$ & $\checkmark$ & $\checkmark$ \\
\hline $\begin{array}{l}\text { 4. (Medium-) High-Tech FuE / Aufw. FuE Verarb. } \\
\text { Gewerbe }\end{array}$ & In & & & & & $\checkmark$ & $\checkmark$ & $\checkmark$ \\
\hline \multicolumn{9}{|c|}{ Indikatoren vorrangig zur Erfassung von Innovationen im Hightech-Sektor } \\
\hline 1. Öffentliche FuE-Ausgaben / BIP & In & $\checkmark$ & $\checkmark$ & $\checkmark$ & $\checkmark$ & $\checkmark$ & $\checkmark$ & $\checkmark$ \\
\hline 2. Private FuE-Ausgaben / BIP & In & $\checkmark$ & $\checkmark$ & $\checkmark$ & $\checkmark$ & $\checkmark$ & $\checkmark$ & $\checkmark$ \\
\hline 3. Risikokapital in der Frühphase / BIP & In & & & $\checkmark$ & $\checkmark$ & $\checkmark$ & $\checkmark$ & $\checkmark$ \\
\hline 4. Patentanmeldungen (USPTO) / Einwohner & Out & & $\checkmark$ & $\checkmark$ & $\checkmark$ & $\checkmark$ & $\checkmark$ & $\checkmark$ \\
\hline 5. Patentanmeldungen (EPA) / Einwohner & Out & & $\checkmark$ & $\checkmark$ & $\checkmark$ & $\checkmark$ & $\checkmark$ & $\checkmark$ \\
\hline 6. Triadic patent families & Out & & & & & $\checkmark$ & $\checkmark$ & $\checkmark$ \\
\hline 7. New community trademarks & Out & & & & & $\checkmark$ & $\checkmark$ & $\checkmark$ \\
\hline 8. New community design & Out & & & & & $\checkmark$ & $\checkmark$ & $\checkmark$ \\
\hline \multicolumn{9}{|c|}{$\begin{array}{l}\text { Indikatoren zur Erfassung der Innovationsfähigkeit in forschungs- und } \\
\text { nicht-forschungsintensiven Sektoren }\end{array}$} \\
\hline 1. W+T-Absolventen & In & $\checkmark$ & $\checkmark$ & $\checkmark$ & $\checkmark$ & $\checkmark$ & $\checkmark$ & $\checkmark$ \\
\hline 2. Bevölkerung mit tertiärem Bildungsabschluss & In & $\checkmark$ & $\checkmark$ & $\checkmark$ & $\checkmark$ & $\checkmark$ & $\checkmark$ & $\checkmark$ \\
\hline 3. Lebenslanges Lernen & In & $\checkmark$ & $\checkmark$ & $\checkmark$ & $\checkmark$ & $\checkmark$ & $\checkmark$ & $\checkmark$ \\
\hline $\begin{array}{l}\text { 4. KMU mit innerbetrieblicher Innovation - } \\
\text { Verarbeitendes Gewerbe + Dienstleistungssektor }\end{array}$ & In & $\checkmark$ & $\checkmark$ & $\checkmark$ & $\checkmark$ & $\checkmark$ & $\sqrt{2})$ & $\checkmark$ \\
\hline $\begin{array}{l}\text { 5. KMU mit kooperativer Innovation - } \\
\text { Verarbeitendes Gewerbe + Dienstleistungssektor }\end{array}$ & In & $\checkmark$ & $\checkmark$ & $\checkmark$ & $\checkmark$ & $\checkmark$ & $\sqrt{2})$ & $\checkmark$ \\
\hline $\begin{array}{l}\text { 6. KMU als Innovatoren nicht-technologischer } \\
\text { Innovationen }\end{array}$ & $\ln$ & & & & $\checkmark$ & $\checkmark$ & $\checkmark$ & $\checkmark$ \\
\hline $\begin{array}{l}\text { 7. Innovationsaufwendungen / Umsatz des } \\
\text { Verarbeitenden Gewerbes + Dienstleistungssektors }\end{array}$ & In & $\checkmark$ & $\checkmark$ & $\checkmark$ & $\checkmark$ & $\checkmark$ & $\sqrt{ } 2)$ & $\checkmark$ \\
\hline 8. Privater Internetzugang (Breitbandnutzung ab 2005) & In & $\checkmark$ & $\checkmark$ & $\checkmark$ & $\checkmark$ & $\checkmark$ & $\checkmark$ & $\checkmark$ \\
\hline 9. Aufwendungen für IKT / BIP & In & $\checkmark$ & $\checkmark$ & $\checkmark$ & $\checkmark$ & $\checkmark$ & $\checkmark$ & $\checkmark$ \\
\hline 10. 20-24-Jährige mit sekundärem Bildungsabschluss & In & & & & & $\checkmark$ & $\checkmark$ & $\checkmark$ \\
\hline 11. Unternehmen mit öffentlicher Innovationsfinanzierung & In & & & & & $\checkmark$ & $\checkmark$ & $\checkmark$ \\
\hline $\begin{array}{l}\text { 12. Marktneuheiten - Verarbeitendes Gewerbe + } \\
\text { Dienstleistungssektor }\end{array}$ & Out & $\checkmark$ & $\checkmark$ & $\checkmark$ & $\checkmark$ & $\checkmark$ & $\sqrt{2})$ & $\checkmark$ \\
\hline $\begin{array}{l}\text { 13. Produkte neu für das Unternehmen, nicht neu } \\
\text { für den Markt }\end{array}$ & Out & & & $\checkmark$ & $\checkmark$ & $\checkmark$ & $\checkmark$ & $\checkmark$ \\
\hline \multicolumn{9}{|c|}{$\begin{array}{l}\text { Aufgelistet sind alle Indikatoren, die im Jahr } 2007 \text { mindestens seit drei Jahren erhoben wurden. Die Indikatoren der Jahre } \\
2005-2007 \text { sind vollständig abgebildet. Die Abkürzungen EPA und USPTO stehen für Europäisches Patentamt und United States } \\
\text { Patent and Trademark Office. } \\
\text { 1) Die Indikatoren werden im Rahmen der Auswertung des EIS in sogenannte Input- und Outputdimensionen unterteilt. Diese Auf- } \\
\text { teilung wurde hier übernommen (Europäische Kommission 2008, S. 35). } \\
\text { 2) Ab } 2006 \text { wurde die Unterteilung in Dienstleistung und Verarbeitendes Gewerbe für diesen Indikator aufgehoben. }\end{array}$} \\
\hline \multicolumn{3}{|c|}{$\begin{array}{l}\text { Quelle: Europäische Kommission 2001: S. 35ff.; 2002: S. 26; 2003b: S. 2; 2004b: S. 28f.; } \\
\text { 2005d: S. 8, 2006: S. 7; 2008: S. } 35 .\end{array}$} & & & & & & \\
\hline
\end{tabular}

der Technologieintensität erscheinen. Das Argument ist hier, dass nicht-forschungsintensive Innovationen eher als inkrementell denn als radikal zu bezeichnen sind (Bender 2005, S. 89). Die Patentierung von inkrementellen Innovationen gestaltet sich aber eher schwierig. Hinzu kommen organisatorische Schwierigkeiten, bei kleinen und mittelgroßen Betrieben oder bei Innovationstätigkeiten, die in Kooperation mit anderen Unternehmen durchgeführt wurden (Kleinknecht et al. 2002). In Deutschland und der EU liegt der Anteil von Patentierungen der Medium-Low- und Lowtech-Sektoren beim Europäischen Patentamt (2003-2005) lediglich bei rund $15 \%$ (OECD 2008b, S. 26).
Die Hälfte der Indikatoren kann den ersten beiden Kategorien (direkter Hightech-Bezug und vorrangig hightechrelevant) zugeordnet werden, was die Schwerpunktsetzung auf die Erhebung von FuEintensiven Innovationen deutlich macht. Das ist nicht weiter überraschend, da die ersten Innovationsanzeiger gezielt auf die Erhebung von Innovationen in HightechSektoren ausgerichtet waren. Diese Sektoren gelten als besonders zukunftsweisend und impulsgebend - auch für Innovationen in nicht-forschungsintensiven Sektoren.

Die Verwendung der sogenannten traditionellen Innovationsindikatoren wie „FuE-Aufwendungen“ und „Patentanmel- dungen“ (vgl. Kleinknecht et al. 2002, S. 110) birgt natürlich Vorteile. Als Grundlage der Analyse besteht hier eine umfangreiche und zuverlässige Datenbasis. Indikatoren wie FuE-Aufwendungen und Patentanmeldungen haben aber den Nachteil, dass sie keine sichere Auskunft über eine erfolgreich abgeschlossene Innovationsgenerierung geben. FuE-Aufwendungen stellen lediglich einen Innovationsinput von vielen dar, der über den Erfolg der Innovation jedoch wenig aussagt. Ähnlich liegt der Fall bei den Patentanmeldungen, da eine Patentanmeldung nicht zwingend das Ergebnis eines Innovationsprozesses sein muss, sondern auch wirtschaftlich ungenutzt bleiben kann. Das heißt, dass bei beiden Indikatoren (FuE-Aufwendungen und Patentanmeldungen) demnach ein Faktor gemessen wird, der nicht zwingend zur Innovation führen muss. Die hier ermittelte hohe Bedeutung von FuE-Tätigkeiten und Patentierungen als Innovationsindikatoren steht allerdings im Widerspruch zur Innovationsdefinition der Kommission, wonach Innovationen immer auch ökonomisch oder gesellschaftlich genutzte Neuheiten sind (Abschnitt 2.1).

Trotz der Dominanz von Hightech(relevanten)-Indikatoren besteht der Innovationsanzeiger aber nicht ausschließlich aus Indikatoren mit einem Bezug zu Hightech-Sektoren. Die in der Kategorie sektorunspezifische Innovationsindikatoren aufgeführten, neuen Innovationsindikatoren (Kleinknecht et al. 2002, S. 113) wie Innovationsausgaben, Markt- und Unternehmensneuheiten haben den Vorteil, dass zum einen ein Innovationsinput über FuETätigkeiten hinaus erhoben werden kann und dass zum anderen die Innovation selbst auch tatsächlich erfassbar ist. Das Problem liegt allerdings in der Verlässlichkeit der Daten, da die Unternehmen selbst darüber befinden, was als Innovation definiert wird und welches Produkt somit innovativ ist. Auch liegen nicht alle notwendigen Daten, wie beispielsweise explizit ausgewiesene Innovationsausgaben, bereit. Diese sektorunspezifischen Indikatoren beinhalten aber Potenzial für Ausdifferenzierungen und Verbesserungen, wie der Vorschlag von Kleinknecht et al. (2002, S. 114) zeigt, über den Indikator „Innovationsausgaben" mehr über den effizienten Einsatz von FuE im Innovationsprozess zu erfahren. Erweiterungsbedarf besteht bei den sektorunspezifischen Innovationsindikatoren, da es hier nur zwei gibt, die das 
eigentliche Ergebnis des Innovationsprozesses erfassen: Marktneuheiten und Unternehmensneuheit. Sinnvoll wäre eine Erweiterung dieser Indikatoren um organisatorische und technische Prozessinnovationen, um auch hier Innovationstätigkeiten erfassen zu können. Diese können erstens hoch innovativ für das Unternehmen oder den Markt sein und zweitens die Wettbewerbsposition des Unternehmens nicht nur durch Effizienzsteigerungen, sondern auch durch damit verbundene Verbesserungen der Produkteigenschaften weiter stärken.

\section{1 \\ Die im Schatten sieht man nicht}

Eine starke Ausrichtung auf Forschung und Entwicklung konnte sowohl bei der Analyse innovationsprogrammatischer Dokumente als auch bei der Analyse der Innovationsindikatoren festgestellt werden. Mit einer derartigen Gewichtung auf Forschungstätigkeiten ist verbunden, dass vor allem forschungsintensive Sektoren und Unternehmen als zukunftsfähig angesehen werden und von eben diesen Politiken profitieren. Zwar werden Diffusionsprozesse in Betracht gezogen, es wird jedoch weitestgehend außer Acht gelassen, dass Diffusion von Innovationen oder innovationsrelevantem Wissen nicht unidirektional von Hightech- in Lowtech-Sektoren, sondern durchaus auch wechselseitig verläuft. Neben dem Argument, dass Lowtech-Unternehmen nicht ausschließlich die Position des Empfängers (Abnehmer innovativer Produkte) einnehmen, wird die Rolle des Empfängers in solchen Diffusionsprozessen des Weiteren auch nur unzureichend betrachtet. Diesbezüglich argumentieren Robertson et al., dass stabile und etablierte „Empfänger-Sektoren“ volkswirtschaftlich von hoher Bedeutung sind, um die Nachfrage für innovative Produkte und Unternehmen und somit die Finanzierung getätigter FuE-Investitionen auch mittelfristig sicherzustellen. (Robertson et al. 2005, S. 134ff.) Ein Beispiel hierfür wäre der Maschinenbau (Medium-Hightech), dessen Kunden im Metallverarbeitenden Sektor (Medium-Lowtech) die Empfänger von oftmals speziell entwickelten Maschinen und Anlagen sind. Zur erfolgreichen Zusammenarbeit bedarf es sogenannter „receptive capacities“ (Abschnitt 2.3), deren eingehendere Betrachtung und gezielte Förderung jedoch durch den politischen Bias auf Forschungsförderpolitik vernachlässigt wird.

Vor allem nicht-forschungsintensive Innovationen, die ähnlich wie radikale Innovationen eine große Auswirkung auf die Wettbewerbsposition des innovierenden Unternehmens haben können (Bender 2005, S. 89), liegen als Folge der Fokussierung auf FuE als zentralen Innovationsinput außerhalb des innovationspolitischen Blickfeldes. Mit dem Konzept der „architectural innovation“ (Henderson/ Clark 1994, S. 10ff.) können derartige Innovationsprozesse treffend beschrieben werden. Kern des Konzepts ist die Umsetzung einer Neuerung durch die Rekombination der bereits bestehenden Komponenten eines technischen Artefakts, dessen grundlegendes Designkonzept jedoch erhalten bleibt. Als Beispiel ziehen Henderson und Clark die Entwicklung eines kleineren Tischventilators heran. Der Tischventilator unterscheidet sich vom Ursprungsmodell, dem Deckenventilator, nicht durch das Design, sondern durch die Anordnung der Komponenten. Es gibt nach wie vor einen Motor und Rotorenblätter, allerdings wurde die Position der Komponenten verändert. Diese Änderung konnte jedoch nur auf Basis neuen, innovativen Wissens über die Architektur, also den Zusammenhang der Komponenten, durchgeführt werden. Begriffe wie radikale und inkrementelle Innovation helfen in diesem Fall nur bedingt weiter, da eine radikale Innovation zwar ähnlich positive ökonomische Auswirkungen hat, aber wohl eher die Erfindung der Klimaanlage anstatt der Weiterentwicklung eines Ventilators wäre. Als inkrementell würde man vielmehr die Größenveränderung eines Ventilators oder den Austausch der Plastikrotoren durch Aluminium zur Stabilitätsverbesserung bezeichnen. Ein prominentes Beispiel für nicht-forschungsintensive Innovationen ist die Entwicklung und Durchsetzung des Transportcontainers in den 1950er Jahren, der das Transportwesen grundlegend veränderte und dessen Effizienz enorm steigerte. Aufgrund der Normung der Container konnten diese auf Schiffen, Zügen und LKWs transportiert werden, ohne dass einzelne Waren mehrfach umgepackt oder verladen werden mussten. Neue wissenschaftliche Erkenntnisse spielten bei der Entwicklung dieser Innovation keine Rolle, dennoch re- volutionierte die Etablierung des Transportcontainers das Transportwesen und noch heute sind die organisatorischen und ökonomischen Auswirkungen sowie weitere, daran anknüpfende technische Innovationen in Häfen wie Hamburg oder Rotterdam tagtäglich zu beobachten. Ähnliche Fälle nicht-forschungsintensiver Innovationen, wie beispielsweise die Entwicklung von Spezialrohren, konnten im Rahmen des Forschungsprojektes PILOT6 ${ }^{6}$ identifiziert werden (Hirsch-Kreinsen 2008). Hier$\mathrm{zu}$ ist nicht nur spezifisch technisches Wissen notwendig, sondern auch die Fähigkeit, allgemein verfügbares Wissen - beispielsweise über die Eigenschaften bestimmter Stahlgüter - auf das konkrete Innovationsprojekt anzuwenden sowie neues Wissen und gegebenenfalls neue Akteurskonstellationen zu kreieren (s. u. Innovation Enabling Capabilities).

Unterstützt wird dieses Argument durch die ökonomische Bedeutung, die der Lowtech-Sektor nach wie vor in den OECD-Staaten einnimmt (Kaloudis et al. 2005, S. 61f.). In Deutschland liegen Beschäftigung und Wertschöpfung (Abbildungen 2 und 3) zwar unter der im Medium-Hightech-Sektor (Maschinenbau- und Fahrzeugbau), aber auch weit über der des Hightech-Sektors. Interessant ist, dass Beschäftigung und Wertschöpfung bei den Medium-Lowtech-Herstellern von 1995 bis 2005 recht stabil sind. Der HightechSektor kann seit 1995 keinen Beschäftigungszuwachs verzeichnen, auch der Anstieg der Wertschöpfung bleibt hier relativ gering.

Verbunden mit der Fokussierung auf FuE als zentralen Innovationstreiber bestätigt sich das in Abschnitt 2.3 erwähnte Problem, dass weitere Innovationspotenziale, wie etwa spezielle Fähigkeiten, vernachlässigt werden. Bender und Laestadius entwickeln in diesem Zusammenhang ,Innovation Enabling Capabilities“. Eher analytisch als empirisch unterscheiden sie zwischen den beiden Fähigkeiten „transforma-

\footnotetext{
6 PILOT steht für das von der EU mit dem fünften Forschungsrahmenprogramm geförderte Projekt „Policy and Innovation in Low-Tech - Knowledge Formation, Employment and Growth Contributions of the "Old Economy' Industries in Europe". Bei PILOT ging es unter anderem um die quantitative Analyse der volkswirtschaftlichen Bedeutung von Lowtech-Industrien sowie um die qualitative Erforschung von Innovationstätigkeiten im sogenannten Lowtech-Sektor (vgl. PILOT-Project 2006 unter: www. pilot-project.org).
} 


\section{Abb. 2: Beschäftigung im Verarbeitenden Gewerbe in Deutschland}

nach Technologieintensität - Beschäftigte in Tsd. -
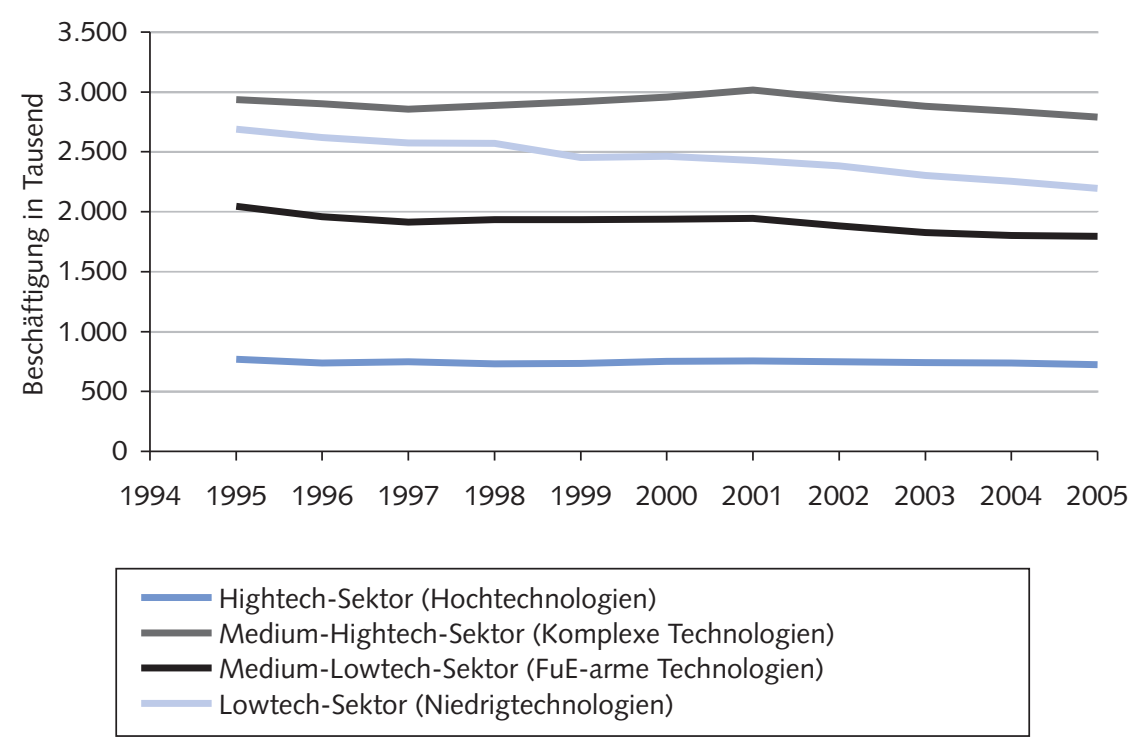

WSI MITTELUNGEN

\section{Abb. 3: Wertschöpfung (zu aktuellen Preisen) im Verarbeitenden Gewerbe in Deutschland nach Technologieintensität 1995-2005 - in Mrd. Euro -}
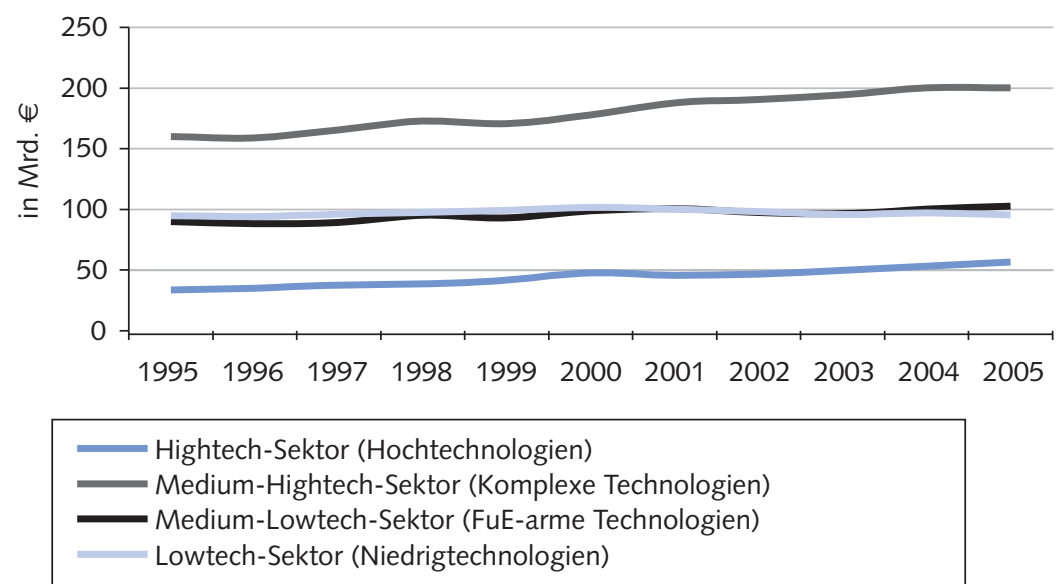

tive“ und „configurational capabilities“ (Bender/Laestadius 2005, S. 136ff.). Sie beschreiben Erstgenannte als Fähigkeit, „to transform available general knowledge and competence into plant, firm or task specific knowledge and competence" sowie Letztere „to synthesise novelty by creating new configurations of knowledge, artefacts and actors" (ebd., S. 136ff.). Als Beispiel führen sie unter anderem das Schweißen von Stahl an, wobei die generelle Fähigkeit, Stahl zu schweißen, zu einer qualitativ ternehmen und Industrien auf verschiedene Akteure, Technologien und Industrien verteilt ist. Eine Wissensbasis besteht aus unterschiedlichen Arten: externes relevantes Wissen wie implizites und kodifiziertes Wissen, Wissen, das in Maschinen inkorporiert ist oder durch wissenschaftliche und technische Literatur, Beratung und Bildung übermittelt wird (Smith 2002, S. 15f.; Bender 2005, S. 93). Die Schwierigkeit der innovativen Tätigkeiten liegt demnach in der Entwicklung spezieller ,innovation enabling capabilities" wie beispielsweise Identifikation, Umgang und Weiterentwicklung von Wissen aus verteilten Wissensbasen und deren unterschiedlichen Arten von Wissen.

\section{Resümee}

Die Konkretisierung und kritische Auseinandersetzung mit der Lissabon-Strategie zeigt, dass sie innerhalb der ersten fünf Jahre ein Verständnis von Innovation etabliert hat, bei dem Forschungs- und Entwicklungstätigkeiten ein zentrales, wenn nicht das zentrale Element des Innovationsprozesses darstellen. Zwar ist die innovationspolitische Agenda interdisziplinär und sehr breit angelegt, dennoch finden sich in den meisten Politikbereichen vorrangig Maßnahmen zur gezielten Förderung von Forschungs- und Entwicklungstätigkeiten. Dieses Innovationsverständnis kann ebenfalls bei den Analysen des Europäischen Innovationsanzeigers identifiziert werden der Datengrundlage, auf die sich die innovationspolitische Programmatik stützt. Aus dieser einseitigen Fokussierung auf FuE als zentralem Innovationstreiber resultiert das Problem, dass nicht-forschungsintensive Innovationen, insbesondere in den sogenannten Lowtech-Sektoren, nur unzureichend oder gar nicht erfasst und betrachtet werden. Eine systematische Förderung von Innovationen und der dazu notwendigen Ressourcen und Kapazitäten in diesen Sektoren bleibt somit aus. Des Weiteren nehmen Lowtech-Unternehmen, deren Anteil an Beschäftigung und Wertschöpfung seit Jahren konstant über dem des HightechSektors liegt, als Abnehmer von Innovationen eine zentrale Position bei der Refinanzierung von Innovationsinvestitionen der Hightech-Unternehmen ein. Die Funktion etablierter Industrien reduziert sich aller- 
dings nicht nur auf die als Abnehmer; sie fungieren auch als Ideengeber für Innovationen (Robertson et al. 2005). Daraus resultiert für Wissenschaft und Politik weiterer Handlungsbedarf, um die Lissaboner Ziele für Wachstum und Beschäftigung zu realisieren. Die Beantwortung verschiedener, noch offener Fragen führt zur Klärung des notwendigen Handlungsbedarfs: Wie können sektorübergreifende Innovationstätigkeiten, die nicht der Logik eines unidirektionalen Technologietransfers „High-toLowtech" entsprechen, gefördert werden? Inwiefern kann Politik die notwendigen
Fähigkeiten und Ressourcen in Hightechund Lowtech-Sektoren fördern? Ähnlich schwierig gestaltet sich die Problematik bei den Innovationsindikatoren: Wie können Daten über Fähigkeiten wie die von Bender/Laestadius (2005) identifizierten „Innovation Enabling Capabilities" erhoben werden, die neben FuE eine zentrale Form der Wissenserzeugung und des Wissensinputs in Innovationsprozesse darstellen? Ein erster Schritt wäre, sich von der Vorstellung zu lösen, dass neues wissenschaftliches Wissen der einzig zentrale Innovationsinput sei. Stattdessen wäre etwa zu fra- gen, woher bei Innovationsprozessen das relevante Wissen stammt, wie Unternehmen dieses in der Regel verteilte Wissen identifizieren und wie sie mit den unterschiedlichen Wissenstypen umgehen, um daraus neues Wissen zu generieren. Dazu wurden hier erste konzeptionelle Überlegungen vorgestellt; weitere gilt es vor allem im Hinblick auf den nach wie vor hohen Anteil an Wertschöpfung, Beschäftigung und Innovativität der etablierten LowtechSektoren zu entwickeln. 


\section{LITERATUR}

Bender, G. (2005): Innovation in Low-Tech Companies - Towards a Conceptualisation of Non-Science-Based Innovation, in: Hirsch-Kreinsen, H./ Jacobson, D./Laestadius, S. (Hrsg.): Low-tech Innovation in the Knowledge Economy, Frankfurt/M., S. 85-98

Bender, G./Laestadius, S. (2005): Non-science based innovativeness. On capabilities relevant to generate profitable novelty, in: Bender, G./Jacobson, D./Robertson, P. (Hrsg.): Perspectives on Economic Political and Social Integration, in: Journal for Mental Changes, Special Edition 1-2, S. 123-170

Europäische Kommission (2001): Arbeitsdokument der Kommissionsstellen - Innovationsanzeiger 2001, SEK(2001) 1414, Brüssel

Europäische Kommission (2002): Arbeitspapier der Bediensteten der Kommission - Europäischer Innovationsanzeiger 2002, SEK(2002) 1349, Brüssel

Europäische Kommission (2003a): Mitteilung der Kommission an den Rat, das Europäische Parlament, den Wirtschafts- und Sozialausschuss der Europäischen Gemeinschaft und den Ausschuss der Regionen - Innovationspolitik: Anpassung des Ansatzes der Union im Rahmen der LissabonStrategie, KOM(2003) 112 endgültig, Brüssel

Europäische Kommission (2003b): 2003 European Innovation Scoreboard: Technical Paper 1 - Indicators and Definitions, erstellt von Hollanders, H., MERIT, Brüssel

Europäische Kommission (2004a): Die Herausforderung annehmen - die Lissabon-Strategie für Wachstum und Beschäftigung, Bericht der hochrangigen Sachverständigengruppe unter Vorsitz von Wim Kok, Luxemburg

Europäische Kommission (2004b): Commission Staff Working Paper European Innovation Scoreboard 2004: Comparative Analysis of Innovation Performance, SEC(2004) 1475, Brüssel

Europäische Kommission (2004c): Exploring Innovation Performances by Sectors, Brüssel

Europäische Kommission (2005a): Mitteilung für die Frühjahrstagung des Europäischen Rates - Zusammenarbeit für Wachstum und Arbeitsplätze. Ein Neubeginn für die Strategie von Lissabon, KOM(2005) 24, Brüssel

Europäische Kommission (2005b): Mitteilung der Kommission an den Rat und das Europäische Parlament - Gemeinsame Maßnahmen für Wachstum und Beschäftigung: Das Lissabon-Programm der Gemeinschaft, $\operatorname{KOM(2005)} 330$ endg., Brüssel

Europäische Kommission (2005c): Integrierte Leitlinien für Wachstum und Beschäftigung (2005-2008), Luxemburg

Europäische Kommission (2005d): European Innovation Scoreboard 2005 - Comparative Analysis of Innovation Performance, Brüssel
Europäische Kommission (2006): European Innovation Scoreboard 2006 - Comparative Analysis of Innovation Performance, Brüssel Europäische Kommission (2008): European Innovation Scoreboard 2007 - Comparative Analysis of Innovation Performance, Brüssel

Europäischer Rat (2000): Schlussfolgerungen des Vorsitzes - Europäischer Rat (Lissabon) 23. und 24. März, o. O.

Europäischer Rat (2002): Schlussfolgerungen des Vorsitzes - Europäischer Rat (Barcelona) 15. und 16. März, SN 100/02, Brüssel

Europäischer Rat (2005): Schlussfolgerungen des Vorsitzes - Europäischer Rat 22. und 23. März, 7619/1/05 REV 1, Brüssel

Grupp, H./Mogee M. E. (2004): Indicators for national science and technology policy: how robust are composite indicators?, in: Research Policy 33, S. 1373-1384

Henderson, R./Clark, K. B. (1990): Architectural Innovation: The Reconfiguration of Existing Product Technologies and the Failure of Established Firms, in: Administrative Science Quarterly 35, S. 9-30

Hirsch-Kreinsen, H. (2008): "Low-Technology": A Forgotten Sector in Innovation Policy; in: Journal of Technology Management \& Innovation 3, S. 11-19

Kaloudis, A./Sandven, T./Smith, K. (2005): Structural Change, growth and innovation: The roles of medium and low tech industries, 19802002, in: Bender, G./Jacobson, D./Robertson, P. (Hrsg.): Perspectives on, Economic Political and Social Integration, in: Journal for Mental Changes, Special Edition 1-2, S. 49-73

Kleinknecht, A./Montfort, V. K./Brouwer, E. (2002): The Non-Trivial Choice between Innovation Indicators, in: Economics of Innovation and New Technology 2, S. 109-121

OECD (2005): Oslo Manual - Guidelines for collecting and interpreting innovation data, $3^{\text {rd }}$ edition, OECD Paris

OECD (2007): Science, Technology and Industry Scoreboard 2007, OECD Publishing, Paris

OECD (2008a): STAN Databaase for Structural Analysis, Internetressource: http://stats.oecd.org/wbos/Index.aspx?DatasetCode= STANO8BIS\&lang=en, Stand: 19.11.2008

OECD (2008b): The OECD Compendium of Patent Statistics 2008, prepared by H. Dernis, OECD

Robertson, P./Pol, E./Carroll, P. (2005): Receptive Capacity of Established Industries as a Limiting Factor in the Economy's Rate of Innovation, in: Hirsch-Kreinsen, H./Jacobson, D./Laestadius, S. (Hrsg.): Low-tech Innovation in the Knowledge Economy, Frankfurt/M.

Smith, K. (2002): What is the "knowledge economy"? Knowledge-intensive industries and distributed knowledge-bases. UNU-INTECH Discussion Paper DP 2002-6 